\section{Emergency resection of a carinal adenoma}

\section{Ganesh Shanmugam Keith Buchan}

J R Soc Med 2006;99:38-39

CARDIOTHORACIC SECTION, 26 NOVEMBER 2004

Emergency carinal resection is a daunting and infrequently performed procedure.

\section{CASE HISTORY}

A man aged 46 developed acute stridor and fibreoptic bronchoscopy showed a large pedunculated mass at the carina. The right main bronchus was completely obstructed and the left main bronchus about $50 \%$ obstructed. Biopsies were non-diagnostic. CT showed the mass to occupy the distal tracheal lumen and there was a large posterior extrabronchial component (Figure 1). The posterior tracheal wall bisected the tumour mass. Endobronchial resection, attempted via a rigid bronchoscope, caused moderate bleeding into the distal airways and the procedure was terminated. Over the next two days ventilation became increasingly difficult, high inflation pressures being required to achieve satisfactory tidal volumes and adequate oxygenation. A decision was made to attempt carinal resection through a right thoracotomy; on left lateral positioning, however, he could not be ventilated satisfactorily and inflation pressures and $\mathrm{pCO}_{2}$ rose. Therefore cardiopulmonary bypass was established by femoro-atrial cannulation. After median sternotomy the superior mediastinal structures were dissected out and the anterior pericardium was mobilized as a pedicle with a broad base of attachment to the aortic arch. The trachea was incised just above the carina to expose the endotracheal tumour (Figure 2). The posterior component arising from the posterior tracheal wall and the adjacent main bronchi was excised with a circumferential margin of normal tracheal wall. The pericardial flap was rotated to bring its serosal surface into the tracheal lumen, and was sutured to the margins of the resulting defect in the posterior wall of the trachea and principal bronchi. Completion bronchoscopy showed widely patent distal trachea and principal bronchi. Mechanical ventilation had to be continued for

Department of Cardiothoracic Surgery, Aberdeen Royal Infirmary, Aberdeen AB25 2ZN, Scotland, UK

Correspondence to: Mr Ganesh Shanmugam, Department of Cardiothoracic Surgery, Western Infirmary, Glasgow G11 6NT, Scotland, UK

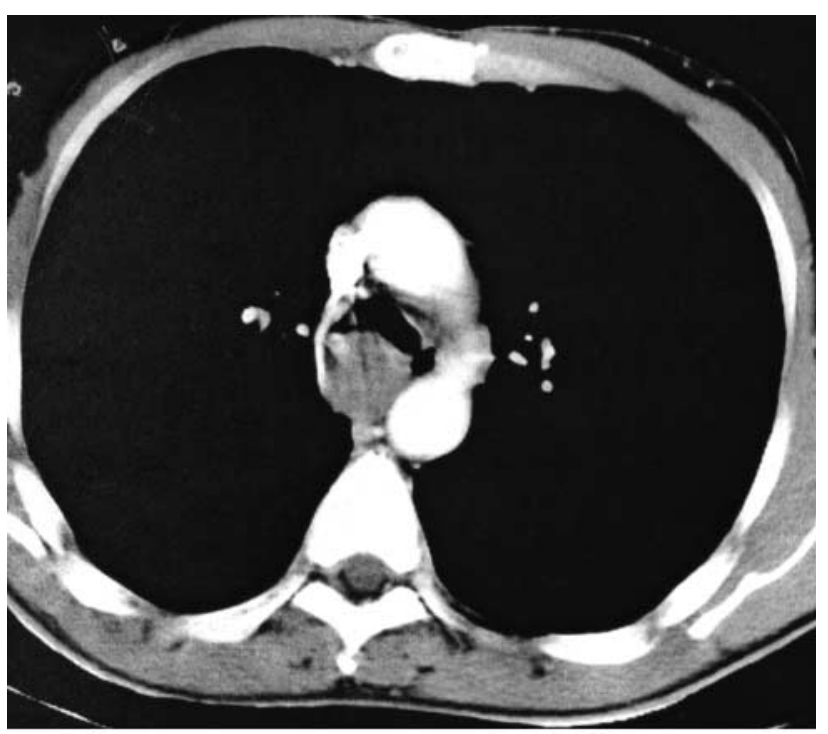

Figure 1 CT of chest showing large tumour arising from the posterior tracheal wall close to the carina

seven days because of non-cardiogenic pulmonary oedema. Three weeks postoperatively he underwent bronchoscopy and dilatation of a mild anastomotic stricture.

After another four weeks he was discharged home. The final pathological diagnosis was pleomorphic adenoma with apparently complete resection. Over the ensuing four months he required three further tracheal dilatations, which were increasingly difficult. He then underwent permanent stenting of the left main bronchus and distal trachea with two Gianturco non-covered wire stents. 2 years later he had no stridor and was symptom-free.

\section{COMMENT}

The most common cause of tracheal or carinal obstruction is lung cancer. When there are signs of respiratory failure,

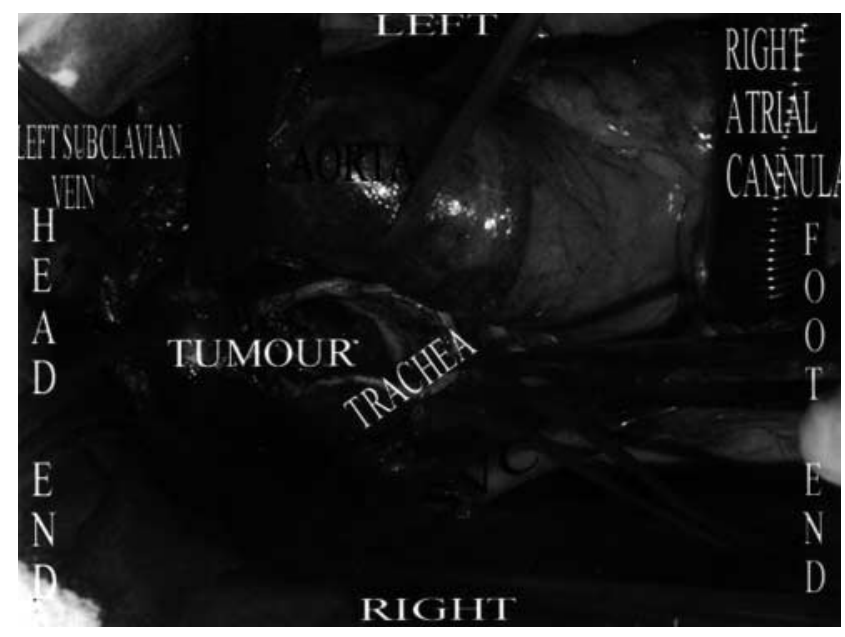

Figure 2 Operative photographs depicting the endoluminal and the extratracheal components (forceps), of the tumour bisected by the posterior tracheal wall; the aorta, innominate vein, superior vena cava and the right pulmonary artery have been taped 
the priorities are to restore adequate ventilation and obtain a pathological diagnosis. ${ }^{1}$

The choice of bronchoscopic techniques includes NdYAG laser, diathermy and cryotherapy, ${ }^{2}$ all of which carry a risk of bleeding. Thereafter, complete resection with primary reconstruction of the airways offers the best chance of cure. Anaesthesia presents special difficulties in these cases. High-frequency jet ventilation can be a useful adjunct. In our patient the ventilatory difficulties were unsurmountable and cardiopulmonary bypass was necessary.

The stricture that developed postoperatively, caused by fibrosis of the pericardial patch, is a well documented complication. Laser and balloon dilatation offer immediate relief, but in cases of recurrence an endobronchial stent can provide good function in the long term.

In the UK, most tracheal and carinal surgery is undertaken in a few specialized centres and, even in these,

\section{Retroperitoneal fibrosis mimicking prostate cancer}

\section{Shabbir ${ }^{1} \quad J$ S Gelister ${ }^{2}$}

J R Soc Med 2006;99:39-40

Retroperitoneal fibrosis typically affects the region extending from the second lumbar vertebra to the pelvic brim. Where it extends below the pelvic brim, encasement of individual pelvic organs can mimic malignant disease. ${ }^{1,2}$

\section{CASE HISTORY}

A previously fit and well retired carpenter aged 65 was investigated after reporting painless macroscopic haematuria of short duration. The only noteworthy physical finding was a firm irregular prostate clinically compatible with a T3/T4 malignant lesion. Abdominal ultrasound showed moderate to gross bilateral hydronephrosis. CT confirmed the hydronephrosis and the irregularity of the prostate (Figure 1); in addition, $\mathrm{CT}$ and chest radiographs revealed extensive bilateral calcified pleural plaques typical of asbestos exposure. Prostate specific antigen was normal $(1.5 \mathrm{ng} /$ $\mathrm{mL}$ ); serum creatinine was raised at $172 \mu \mathrm{mol} / \mathrm{L}$ and the erythrocyte sedimentation rate (ESR) was $56 \mathrm{~mm} / \mathrm{h}$.

${ }^{1}$ Department of Urology, St George's Hospital, London SW17 ORE; ${ }^{2}$ Barnet General Hospital, London, UK

Correspondence to: M Shabbir

E-mail: majidshabbir@hotmail.com the large majority of resectional procedures are done electively, in a controlled environment. However, any cardiothoracic centre may find itself confronted with one of these emergencies; and if transfer is too dangerous to the patient, the local surgeons may have to rely on basic principles and their trade craft. In the instance described here, they were much helped by the availability of cardiopulmonary bypass.

\section{REFERENCES}

1 Venuta F, Rendina EA, De Giacomo T, et al. Nd:YAG laser resection of lung cancer invading the airway as a bridge to surgery and palliative treatment. Ann Thorac Surg 2002;74:995-8

2 Daddi G, Puma F, Avenia N, et al. Resection with curative intent after endoscopic treatment of airway obstruction. Ann Thorac Surg 1998;65:203-7

3 Burns KE, Orons PD, Dauber JH, et al. Endobronchial metallic stent placement for airway complications after lung transplantationlongitudinal results. Ann Thorac Surg 2002;74:1934 41

On examination under anaesthesia a distinct pelvic mass was felt at the level of the bladder base; the bladder was cystoscopically normal. Retrograde imaging showed bilateral tapering strictures of the distal ureters suggestive of external compression. Ureteric stents were inserted on both sides. Biopsies from the pelvic lesion contained dense fibrous tissue compatible with retroperitoneal fibrosis; no evidence of malignant disease was found in the pelvic mass or the prostate. Cancer having been excluded, the patient was started on prednisolone. Four and eight months later, retrograde studies showed only marginal improvement in the ureteric obstruction despite a fall in the ESR to

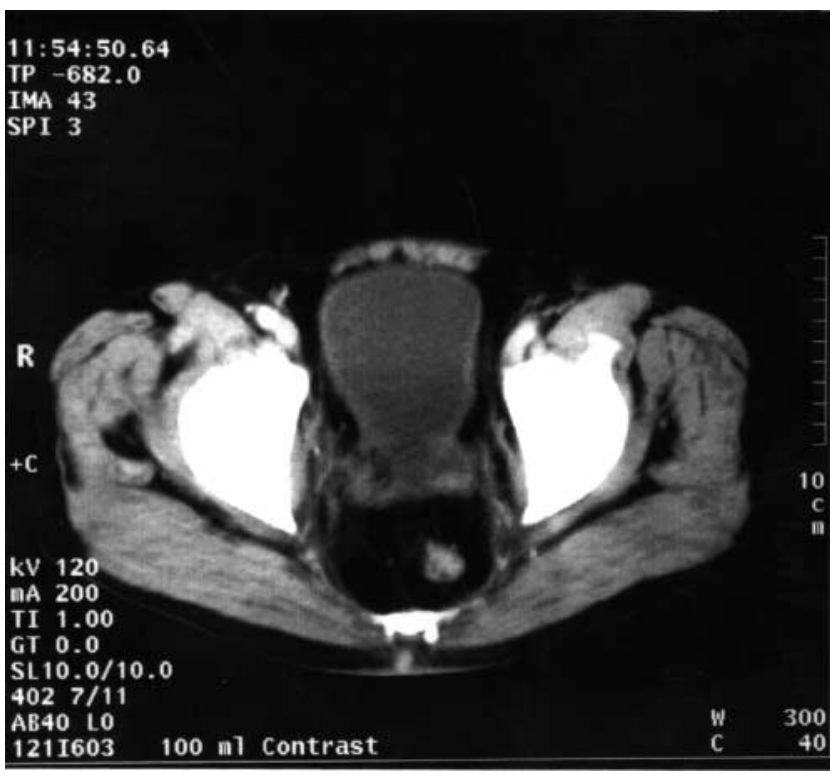

Figure 1 CT of pelvis 